Our Nature (2007)5: 60-66

\title{
Some Euglenophycean Algae from Biratnagar, Nepal
}

\author{
S.K. Rai ${ }^{1}$ and R.K. Rai ${ }^{2}$ \\ ${ }^{I}$ Department of Botany, P.G.Campus, T.U., Biratnagar, Nepal \\ ${ }^{2}$ Department of Botany, M.M.A.M Campus, T.U., Biratnagar, Nepal
}

Received: 23.10.2007, Accepted: 15.12.2007

\begin{abstract}
A total 19 euglenophycean taxa (Euglena 4 , Phacus 8, Trachelomonas 5, Lepocinclis 2 ) has been reported from ponds, pools and ditches of Biratnagar in the present communication. Among these, nine taxa viz., Euglena sanguinea Ehrenberg, E. tripteris (Dujardin) Klebs, Phacus birgei Prescott, P. helikoides Pochmann, P. pleuronectes (Mueller) Dujardin, P. spirogyra Drezepolski var. maxima Prescott, Trachelomonas armata (Ehrenberg) Stein var. steinii Lemmermann, T. similis Stokes and Lepocinclis ovum (Ehrenberg) Lemmermann are being reported for the first time from Nepal.

Keywords: Algae, Euglenophyceae, Phacus, Biratnagar, Nepal.
\end{abstract}

\section{Introduction}

The euglenophycean flora of Nepal is poorly studied as no sufficient literature available. Hirano (1963), Hickel (1973a,b) and Ishida (1986) have described few taxa while studying other groups of algae. Sahay et al. (1953) have reported ten taxa (Phacus 4, Trachelomonas 5, Lepocinclis 1) from Chandranigahpur and Hetauda. Das and Verma (1996) have also collected three taxa from Budhi Khola, Tandi and Khageri Tal, Tikauli, Chitwan. Similarly, Habib (1997) has identified eight taxa of Euglena from Mahendranagar, Kanchanpur along with other algae. The major contribution on the euglenophycean flora of Nepal was made by Ioriya (1988) who has recorded 25 taxa (Euglena 8, Phacus 6, Trachelomonas 10, Petalomonas 1) from Kathmandu valley. Thus, a total 56 euglenophycean taxa has been recorded from this country.

Biratnagar is an industrial city located at Lat. $26^{\circ} 20^{\prime} \mathrm{N}$ and Long. $87^{\circ} 16^{\prime} \mathrm{E}$ in the terai plain of eastern Nepal at an elevation of 72 $\mathrm{m}$ amsl. It has a tropical monsoonic climate with $41^{\circ} / 4^{\circ} \mathrm{C}$ max./min. temperature and $1730.2 \mathrm{~mm}$ average annual rainfall. The hot and humid climate and slightly polluted lentic habitats influence the luxuriant growth of euglenoids in this area. Since there has been no report on euglenophycean algae from eastern Nepal, an attempt was made to describe the algae.

\section{Materials and methods}

Samples were collected from ponds, pools and ditches during 9-11am with the help of plankton net (mesh size $0.5 \mathrm{~mm}$ ) and preserved in $4 \%$ formalin. For examination, both fresh and preserved samples of each collection were pipetted separately to the glass slides, covered with cover slips and observed thoroughly under microscope. Illustrations of all the taxa studied were made with the help of camera lucida or by free hand drawings. All the collections are deposited in the herbarium of the Botany Departement, P.G. Campus, Biratnagar. 
S.K. Rai and R.K. Rai / Our Nature (2007)5: 60-66

\section{Taxonomic description}

Division: Euglenophyta

Class: Euglenophyceae

Order: Euglenales

Family: Euglenaceae

Genus: $\quad$ Euglena Ehrenberg 1838

\section{Euglena acus Ehrenberg (Fig. 1)}

Fritsch, F.E. 1935, P.726, Figs. a-b; Prescott, G.W. 1951, P.390, Pl. 85, Fig. 28; Tiffany, L.H. and M.E. Britton 1952, P. 321, Pl. 87, Fig. 1003; Ioriya, T. 1988, P. 32, Fig. 6.

Cells elongated, spindle shaped, 140-180 $\mu \mathrm{m}$ long, $10-13 \mu \mathrm{m}$ broad, anterior end narrowed and truncate, posterior end long, fine tapering point; chloroplast numerous, disc like; paramylon bodies several, long rods.

Collection No. and Date: B-51, 19-08-2007.

Locality: Roadside ditches at Navintol.

Distribution: A shallow pool at Dillibazar and Swayambhu, Kathmandu (Hirano, 1963; Ioriya, 1988).

\section{Euglena oxyuris Schmarda (Fig. 2)}

Fritsch, F.E. 1935, P. 726, Fig. 293h; Tiffany, L.H. and M.E. Britton 1952, P. 318, Pl. 87, Fig. 1010.

Cells elongate-cylindric and twisted, 160 $\mu \mathrm{m}$ long, $22 \mu \mathrm{m}$ broad, anterior end round with slight indentation at opening of canal, posterior end taper to short tail piece; periplast with prominent striation; chloroplast numerous, disc like; nucleus centrally located; paramylon bodies 2, anular elongated rings on either side of nucleus.
Collection No. and Date: B-54, 19-08-2007.

Locality: Malaya roadside ditches near P.G. Campus.

Distribution: E. oxyuris var. charkowiensis has been reported from Khaste lake, Kaski (Hickel, 1973a)

3. Euglena sanguinea Ehrenberg (Fig. 11)

Fritsch, F.E. 1935, P. 727, Fig. 240h; Prescott, G.W. 1951, P. 394, Pl. 86, Figs. 12; Tiffany, L.H. and M.E. Britton 1952, P. 317, Pl. 87, Fig. 1013.

Cells ovoid, pyriform to sub-cylindric, 24$33 \mu \mathrm{m}$ broad, 55-120 $\mu \mathrm{m}$ long, anterior end narrow rounded, posterior end taper to a short blunt tail piece; periplast spirally striated; paramylon bodies numerous ovoid grains; hematochrome granules present.

Collection No. and Date: B-60, 25-08-2007.

Locality: Pitchara pond.

Distribution: New record for Nepal.

4. Euglena tripteris (Dujardin) Klebs (Figs. $12,15)$

Prescott, G.W. 1951, P. 394, Pl. 86, Figs. 46; Tiffany, L.H. and M.E. Britton 1952, P. 318, Pl. 87, Fig. 1014.

Cells rigid, elongate cylindric and twisted, 70-80 $\mu \mathrm{m}$ long, 8-15 $\mu \mathrm{m}$ broad, anterior end broadly rounded, posterior end taper to form sharp tip; chloroplast numerous, disc shaped; paramylon bodies 2, thick rods on either side of centrally located nucleus.

Collection No. and Date: B-51, 19-08-2007

Locality: Roadside ditches at Navintol

Distribution: New record for Nepal.

Genus: Phacus Dujardin 1841. 


\section{Phacus birgei Prescott (Fig. 4)}

Prescott, G.W. 1951, P. 398, Pl. 87, Fig. 11.

Cells broadly ovoid, 70-80 $\mu \mathrm{m}$ long, 50-60 $\mu \mathrm{m}$ broad, anterior end broadly round, posterior end produce long, tapering, oblique caudus; periplast very finely striated, margin of the cell sharply notched with 4 small indentations on either side; paramylon bodies one large and numerous small circular plates.

Collection No. and Date: B-55, 20-08-2007.

Locality: Pond at P.G. Campus.

Distribution: New record for Nepal.

6. Phacus curvicauda Swirenko (Fig. 17)

Prescott, G.W. 1951, P. 399, Pl. 88, Fig. 21; Ioriya, T. 1988, P. 35, Fig. 21.

Cells orbiculare to broadly ovoid, $28-35 \mu \mathrm{m}$ long, 24, $26 \mu \mathrm{m}$ broad, anterior end rounded, posterior end broadly rounded with a short curved caudus; periplast longitudinally striated; paramylon body 1 or 2 circular disc.

Collection No. and Date: B-62, 25-08-2007.

Locality: Pitchara pond.

Distribution: Ponds at Pashupatinath, Swayambhu and near Royal Palace (Ioriya, 1988).

7. Phacus helikoides Pochmann (Figs. 8, 13)

Prescott, G.W. 1951, P. 400, P. 87, Fig. 9.

Cells fusiform, twisted throughout the entire length, 70-110 $\mu \mathrm{m}$ long, 32-45 $\mu \mathrm{m}$ broad, anterior end briefly narrow and bilobed, posterior end taper to form spirally twisted, long, straight caudus; periplast longitudinally striated; paramylon body one large circular body.

Collection No. and Date: B-52, 19-08-2007.

Locality: Roadside ditches at Navintol.

Distribution: New record for Nepal.

8. Phacus longicauda (Ehrenberg) Dujardin (Fig. 10)

Fritsch, F.E. 1935, P. 726, Fig. 239i; Prescott, G.W. 1951, P. 400, Pl. 87, Fig. 1; Tiffany, L.H. and M.E. Britton 1952, P. 323, Pl. 88, Fig. 1022; Ioriya, T. 1988, P. 35, Fig. 24.

Cells ovoid, flat, 85-170 $\mu \mathrm{m}$ long, 45-70 $\mu \mathrm{m}$ broad, anterior end broadly rounded, posteriorly taper gradually to form a long straight and sharply pointed caudus; periplast longitudinally striated; paramylon body 1 , large circular plate.

Collection No. and Date: B-53, 19-08-2007.

Locality: Roadside ditches at Navintol.

Distribution: A pond at Kathmandu (Ioriya, 1988); Fish pond at Hetauda (Sahay et al., 1993).

9. Phacus pleuronectes (Muell.) Dujardin (Fig. 16)

Prescott, G.W. 1951, P. 402, Pl. 88, Fig. 16; Tiffany, L.H. and M.E. Britton 1952, P. 323, Pl. 88, Fig. 1019.

Cells broadly ovoid, 40-80 $\mu \mathrm{m}$ long, 28-50 $\mu \mathrm{m}$ broad, slightly twisted, posterior caudus short stout and obliquely turned; periplast longitudinally striated; paramylon body 1 , ring like disc.

Collection No. and Date: B-61, 25-08-2007.

Locality: Roadside ditches at Pitchara. 
Distribution: New record for Nepal.

10. Phacus pseudoswirenkoi Prescott (Fig. 5)

Prescott, G.W. 1951, P. 402, Pl 85, Fig. 26; Pl. 87, Fig. 2; Pl. 88, Fig. 14.

Cells orbicular, $41 \mu \mathrm{m}$ long, $30 \mu \mathrm{m}$ broad, posterior caudus short, sharp and curved, anterior end broadly rounded; periplast longitudinally striated, with medianly located lateral notch on the right side; paramylon body single, large circular disc.

Collection No. and Date: B-63, 25-08-2007.

Locality: Pitchara pond.

Distribution: Kara river, Hetauda (Sahay et al., 1993)

11. Phacus spirogyra Drezepolski var. maxima Prescott (Fig. 3)

Prescott, G.W. 1951, P. 403, Pl. 87, Figs. 46.

Cells ovoid to somewhat oblong, 80-115 $\mu \mathrm{m}$ long, 40-45 $\mu \mathrm{m}$ broad, anteriorly rounded with a prominent median protrusion, abruptly narrowed posteriorly into a long caudus; periplast longitudinally striated with spiral row of granules; paramylon bodies 2, large elongated rings.

Collection No. and Date: B-52, 19-08-2007.

Locality: Roadside ditches at Navintol.

Distribution: New record for Nepal.

12. Phacus suecicus Lemmermann (Fig. 6)

Prescott, G.W. 1951, P. 403, Pl. 88, Figs. 2-3.

Cells broadly ellipsoid or ovate, $42 \mu \mathrm{m}$ long, $25 \mu \mathrm{m}$ broad, anterior end truncate with a prominent median papilla, posterior end produce slightly deflected sharp caudus; periplast longitudinally striated with row of sharp granules; chloroplast numerous, circular discs; paramylon bodies 2, lateral land peripheral plates.

Collection No. and Date: B-70, 01-09-2007.

Locality: Pitchara pond.

Distribution: P. suecicus var. inermius has been reported from a pond at Dillibazar, Kathmandu (Hirano, 1963).

Genus: Trachelomonas Ehrenberg 1835.

13. Trachelomonas armata (Ehrenberg) Stein var. longispina (Playf.) Deflandre (Fig. 19)

Prescott, G.W. 1951, P. 411, Pl. 83, Fig. 27.

Test broadly ovate, $40-43 \mu \mathrm{m}$ long, $30 \mu \mathrm{m}$ diameter, with spines; flagellum aperture without collar but with a circle of erect spines at the margin; spines short in anterior region but stout, both short and long in posterior region.

Collection No. and Date: B-62, 25-08-2007.

Locality: Pitchara pond.

Distribution: Kara river, Hetauda (Sahay et al., 1993).

14. Trachelomonas armata (Ehrenberg) Stein var. steinii Lemm. (Fig. 20)

Prescott, G.W. 1951, P. 411, Pl. 83, Fig. 26.

Test broadly ovate, $40 \mu \mathrm{m}$ long, $30 \mu \mathrm{m}$ diameter; flagellum aperture without collar but with circle of erect spines; wall spiny; spines stout and sparsely distributed in anterior and posterior region.

Collection No. and Date: B-53, 19-08-2007.

Locality: Roadside ditches at Navintol.

Distribution: New record for Nepal. 


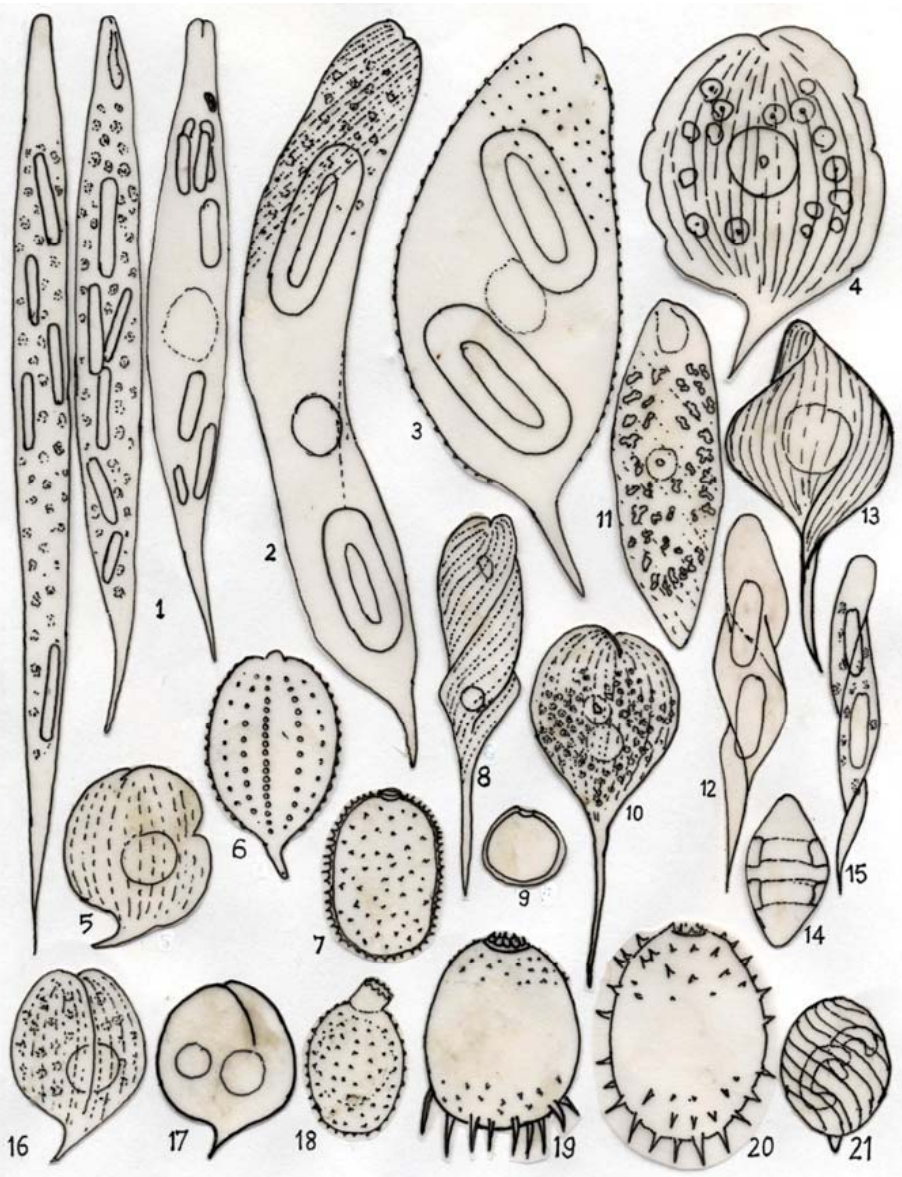

\section{Explanation of figures}

Fig. 1: Euglena acus Ehr. (x850), Fig. 2: Euglena oxyuris Schm. (x950), Fig. 3: Phacus spirogyra Drez. var. maxima Presscott (x950), Fig. 4: Phacus birgei Prescott (x750), Fig. 5: Phacus pseudoswirenkoi Prescott (x945), Fig. 6: Phacus suecicus Lemm. (x900), Fig. 7: Trachelomonas hispida (Perty) Stein (x950), Figs. 8 \& 13: Phacus helikoides Pochmann (x750), Fig. 9: Tracheliomonas volvocina Ehr. (x900), Fig. 10: Phacus longicauda (Ehr.) Duj. (x500), Fig. 11: Euglena sanguinea Ehr. (x750), Figs. 12\&15: Euglena tripteris (Duj.) Klebs (x880), Fig. 13: Phacus helicoids Pochmann (x750), Fig. 14: Lepocinclis fusiformis (Carter) Lemm. (x600), Fig. 16: Phacus pleuronectes (Muell.) Duj. (x500), Fig: 17: Phacus Curvicauda Swir. (x750), Fig. 18: Trachelomonas similis Strok, Fig. 20: Trachelomonas armata (Ehr.) Stein var. steinii Lemm. (x1100) 
S.K. Rai and R.K. Rai / Our Nature (2007)5: 60-66

15. Trachelomonas hispida (Perty) Stein (Fig. 7)

Prescott, G.W. 1951, P. 414, Pl. 83, Fig. 35; Tiffany, L.H. and M.E. Britton 1952, P. 327, Pl. 88, Fig. 1027; Ioriya, T. 1988, P. 33, Fig. 11.

Test ovoid to oblong, $31 \mu \mathrm{m}$ long, $21 \mu \mathrm{m}$ diameter, thickly covered with short spines, collar slightly raised.

Collection No. and Date: B-56, 22-08-2007.

Locality: Pond at Mahendra Morang Campus.

Distribution: Taudaha lake, Kathmandu (Hickel, 1973b); Pool at Balaju, Kathmandu (Ioriya, 1988).

16. Trachelomonas similes Stokes (Fig. 18)

Prescott, G.W. 1951, P. 417, Pl. 84, Fig. 12; Pl. 85, Figs. 10, 13; Tiffany, L.H. and M.E. Britton 1952, P. 327, Pl. 88, Fig. 1031.

Test oblong-ellipsoid, 26-35 $\mu \mathrm{m}$ long, 14-19 $\mu \mathrm{m}$ diameter; wall roughened by irregular shaped granulations, flagellum aperture in a curved collar bearing irregular teeth.

Collection No. and Date: B-53, 19-08-2007.

Locality: Roadside ditches at Navintol.

Distribution: New record for Nepal.

17. Trachelomonas volvocina Ehrenberg (Fig. 9)

Prescott, G.W. 1951, P. 419, Pl. 83, Fig. 1; Tiffany, L.H. and M.E. Britton 1952, P. 326, Pl 88, Fig. 1026; Ioriya, T. 1988, P. 33, Fig. 9.

Test globose, 14-16 $\mu \mathrm{m}$ in diameter, flagellum aperture without a collar, wall smooth, yellowish.
Collection No. and Date: B-56, 22-08-2007.

Locality: Pond at Mahendra Morang Campus.

Distribution: Rupa, Phewa, Begnas and Khaste lakes, Kaski (Hickel, 1973a).

Genus: Lepocinclis Perty 1849

\section{Lepocinclis fusiformis (Carter)} Lemmermann (Fig. 14)

Prescott, G.W. 1951, P. 406, Pl. 89, Figs. 1-4.

Cells broadly fusiforms, $30 \mu \mathrm{m}$ long, $16 \mu \mathrm{m}$ broad; Periplast spirally striated; paramylon bodies 2, circular incomplete rings.

Collection No. and Date: B-52, 19-08-2007.

Locality: Roadside ditches at Navintol.

Distribution: A fish pond at Hedauda (Sahay et al., 1993).

19. Lepocinclis ovum (Ehrenberg) Lemmermann (Fig. 21)

Prescott, G.W. 1951, P. 407, Pl. 89, Figs. 56; Tiffany, L.H. and M.E. Britton 1952, P. 322, Pl. 88, Fig. 1025.

Cells broadly ovate with short, blunt caudus, $30 \mu \mathrm{m}$ long, $21 \mu \mathrm{m}$ broad, rounded both anteriorly and posteriorly; periplast spirally striated; paramylon bodies 2, circular incomplete rings.

Collection No. and Date: B-52, 19-08-2007.

Locality: Roadside ditches at Navintol.

Distribution: New record for Nepal.

\section{Acknowledgements}

The authors are thankful to the Head, Department of Botany, P.G. Campus, T.U., Biratnagar for laboratory facility. 


\section{S.K. Rai and R.K. Rai / Our Nature (2007)5: 60-66}

\section{References}

Das, S.N. and B.N. Verma 1996. Algal flora of Chitwan and Nawalparasi districts of Nepal. Phycos 35 (1-2): 119-127.

Fritsch, F.E. 1935. The structure and reproduction of the algae. Vol. 1, Cambridge University Press. $791 \mathrm{p}$.

Habib, I. 1977. Algal flora from Mahendranagar, Nepal. $J$. Econ. Tax. Bot. 21(1): 19-26.

Hickel, B. 1973a. Limmological investigations in lake of Pokhara valley, Nepal. Int. Rev. ges Hydrobiol. 58(5): 659-672.

Hickel, B. 1973b. Phytoplanktons in two ponds in Kathmandu valley, Nepal. Int. Rev. ges Hydrobiol. 58(6): 835-842.

Hirano, M. 1963. Fresh water algae from the Nepal Himalaya, collected by a member of Japanese
Climbing Expedition. Contr. Biol. Bal; Kyoto University, Japan. 16: 1-23.

Ioriya, T. 1988. Some euglenoid flagellates from Kathmandu. In: Cryptogams of the Himalayas, Vol. 1 (Eds. M. Watanabe and S.B. Malla), National Science Museum, Tsukuba, Japan. pp. 31-37.

Ishida, Y. (Ed.) 1986. Studies on distribution, adaptation and evolution of micro-organisms in Nepal Himalayas (Second report), Kyoto, Japan. pp. 3-13.

Prescott, G.W. 1951. Algae of the western great lakes area. WM.C. Brown Publishers, Dubuque, Iowa. $977 \mathrm{p}$.

Sahay, A.P., P.K. Das and B.N. Verma 1993. Studies on the algal flora of Nepal-II: Cyanophyceae and Euglenophyceae. Geophytology 23(1): 181-183.

Tiffany, L.H. and M.E. Britton 1952. The algae of Illinois. Hafner Publishing Co., New York. 407p. 